\section{Impedance plethysmygraphy and cardiac output}

\author{
E. J. FLANAGAN \\ University of Newcastle-upon-Tyne, England
}

Whenever the heart beats, the volume occupied by the heart changes. Since there is a flow of blood to and from that of the surrounding tissue, as an early warning of cardiac arrest it was thought that an impedance measuring device might have significant advantages. A simple impedance plethysmograph was constructed and proved that signals could be obtained simply by attaching electrodes to the S's chest. These signals were due to the changing resistance in the chest, and, among other signals, there could be found a cyclic change which ceased immediately the heart stopped beating.

Encouraged by these earlier experiments and stimulated by the need to measure respiratory phenomena using an impedance technique, we constructed an improved impedance plethysmograph. This equipment would allow the measurement of any varying resistance within the body, while being completely insensitive to changes in electrode impedance.

When recording respiratory changes, we observed a much smaller amplitude component which varied with the heart rate; this was, at this stage, a slight embarrassment to experiments and so was filtered out and removed.

The idea that the amplitude of this secondary signal might vary with either blood pressure or cardiac output led to the use of a computer of averaged transients to average the complex waveform. This instrument was used initially because it was available rather than because it was ideal for the job. The waveform was obtained by triggering the averaging computer with the $R$ wave of the electrocardiogram and allowing the averaging computer to average over a number of cycles. With a normal $S$ it was quickly found that an averaged trace varied with cardiac output.

As with many biological experiments, it is quite difficult to obtain perfect recordings of the phenomena one is interested in, and it was suggested that, since a number of records were lost due to artifact reaching the averaging computer, it would be much more sensible to allow ourselves to compute a running average.

About this time, a PDP-8/I with laboratory peripheral Type AXO8 became available, and it was a relatively simple step to suggest its use to perform a running average.

The programming of such a problem in PAL III presents no particular difficulties, though some interesting programming points do arise.

The program was arranged so that keyboard control could be exercised over the workings of the program at any time. An outline of the flow diagram for the program is shown in Fig. 1.

Having performed the initial analogue adjustments of the $X-Y$ plotter attached to the computer and typed out the initial operator instructions on the Teletype, from this point we organized on the program by using a control subroutine. The functions available were those of being able to calibrate the internal RC clock of the AXO 8 by means of a numeric display on the computer oscilloscope. Another section set the number of sweeps the program was to compute the average over using the oscilloscope display and one of the analogue potentiometers. A third section constituted the main averaging section with subsidiary display and slow-speed plotting facilities should these be required, in addition to the on-line computed average which is always displayed.

The program uses the DECUS $6 \times 4$ character generator subroutine No. $5 / 8-23$ for all character information to be displayed onto the oscilloscope.
The calibrate clock routine was constructed to measure the time taken for one complete sweep of the oscilloscope, i.e., the time taken to read 512 sample points. This was done by counting the number of crystal clock pulses which occur during 512 RC clock pulses. After this count is made, the relative conversions from binary to decimal are performed and the results displayed for a short period of time on the oscilloscope before the program reenters the calibrate phase. By immediately following the calibrate clock routine by a control subroutine jump and putting the entire system into a closed loop, the program would stay in the calibrate clock position until asked to go elsewhere via the teleprinter and control subroutine.

The subroutine which asked for the number of sweeps over which the record was to be averaged simply used the DECUS message display program, allowing, at the beginning of each display of the message, one of the potentiometers to be read into the computer and divided down into the required range of values, the answer of this division being displayed at the end of the message.

The display and plot subroutines were essentially very similar in that each of them used an autoindex register and displayed via the AXO8 $X-Y$ registers with similar instructions. However, in order to control the plotter and particularly to stop the hunting of the pen of the plotter during the display subroutine, the relay outputs available with AXO8 were used but connected the plotter only during the plot portion of the program. These required that a number of fixed time delays be built into the plot routine to allow the pen recorder to complete its tasks before the computer issued successive runs.

The most interesting part of the

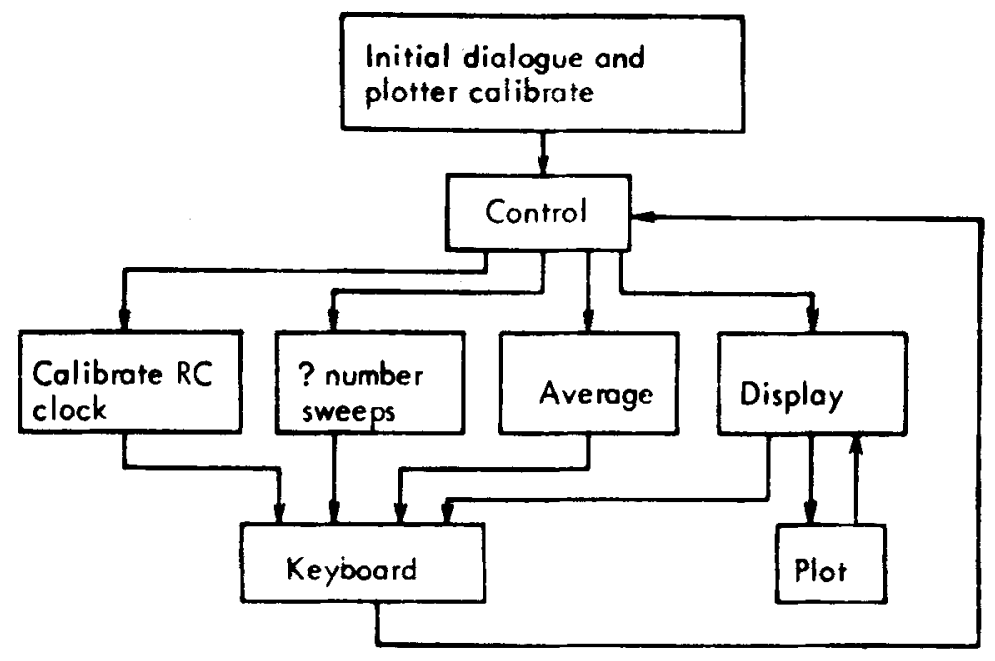

Fig. 1. Average program block diagram. 


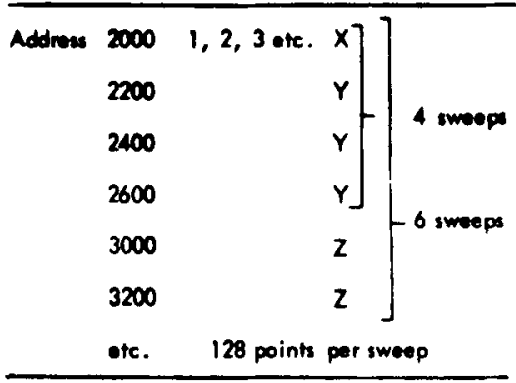

Fig. 2. Allocation of storage for a veraging.

program to run was that of the actual averaging routine. Initially, when this subroutine is entered the computer waits in a trigger loop which demands that a pulse has occurred on one of the Schmitt triggers associated with the AXO8. Once a trigger point is found, as defined by analogue circuits from the $R$ wave of the electrocardiogram, then the RC clock controls the conversions while the program organizes these to be deposited in successive core locations for any one sweep. Successive triggers restart the addressing of these locations so that similar points on successive waveforms occur with similar relationships to the base address of a computer page.

The actual averaging is performed as each point is read by calculating the sum of the values similarly located in the various pages of memory currently in use, i.e., point $\mathrm{X}$ is averaged with all points $Y$ for four sweeps in Fig. 2.

Respiratory rejection is increased by calling the initial value after recording an ECG trigger zero, and subsequently subtracting this value from successive points, thus restoring the trace baseline at the start of each sweep. This means that instead of having to contend with the total variation due to respiration, the averaging program is now simply removing the variable slope of the respiratory trace instead of both the slope and the baseline shift.

After each calculation, the trigger is rechecked to see if there has been a further trigger. If not, the sweep is allowed to continue; if there has been, then a new sweep is started. In this way, a continuous output is obtained of the average of a number of complexes.

To date, the program has allowed us to evaluate the optimal number of sweeps over which to average. This turns out to be about 10 . If one increases significantly the number of sweeps being used, then the averaged waveform is very slow to respond to true variations, but if one decreases the number of sweeps, then the respiration causes the latter portions of each cycle of the signal to become distorted.

The complete evaluation of the impedance cardiogram is progressing at the present moment. We have satisfied ourselves that on various $S s$ the changes in signal level observed from the computer results during patient exercise or following the administration of drugs are very similar to the variations one would expect in cardiac output.

Because of the difficulties of measuring accurately cardiac output in humans (since it is not recognized surgical procedure to access the main aorta to position a flowmeter over it) as yet absolute calibration has not been made.

The other techniques of indirect cardiac output of dye dilution or isotope dilution will not allow their repetition to check for variations in cardiac output. It is therefore imminent that we implant a flowmeter probe over the aorta to allow measurement of beat-by-beat flow. This will be used to check the accuracy to the impedance method for measuring relative flows.

Because of the difficulties in positioning electrodes in an accurate spatial arrangement with respect to the heart itself, it is not expected that impedance techniques will yield an easy way of measuring actual blood flow without some other calibration. What is, however, likely is that impedanee techniques will be used to measure relative cardiac output.

Should this research work come to the expected conclusion, it could well make significant contributions to the anaesthetic management of patients in hospitals in that the effects on any individual's heart of certain drugs could be assessed much earlier in the course of treatment. 